\title{
Responsabilidad social de la Psicología frente a la violencia'
}

Patricia Vaca Vaca² María Clara Rodríguez D. ${ }^{3}$

Resumen

En este artículo se hace un análisis sobre la responsabilidad social de la Psicología frente al fenómeno de la violencia. Se parte de su reconocimiento en este contexto como una de las problemáticas de mayor relevancia y de salud pública, que coincide con la valoración que de ella han hecho estudios realizados a nivel internacional. Se destaca la importancia de la proyección social y la formación de futuros profesionales desde la universidad, así como algunos de los estudios sobre las diferentes expresiones de violencia en este medio. Se asumen como importantes las recomendaciones planteadas en el informe mundial de la OMS sobre violencia y salud, y la compren- 
sión de la realidad de la violencia más allá del ámbito privado e individual en el que priman criterios "curativos" o legales, para reconocerla como un hecho histórico social frente al cual la Psicología debe aportar elementos para la búsqueda de un proyecto ético/social de construcción de sujeto y comunidad. La investigación y praxis soportada en valores solidarios de respeto por el otro y formas diferentes de solucionar el conflicto, serán estrategias que deberán ser sustentadas.

Palabras clave: Responsabilidad social; Psicología; Violencia.

\section{Abstract}

This paper presents an analysis of social responsibility of psychology in relation to the phenomenon of violence. It is based on the recognition of violence as a highly relevant problem, as well as being a public health issue in our context, which coincides with other studies carried out at international level. It highlights the social projection and the importance of training future professionals, starting at university level, as well as some of the studies on different expressions of violence in this country. The World Health Organization's recommendations on violence and health are important, as is the understanding of the reality of violence which reaches beyond the private and individual sphere, where priority is given to 'curative' or legal criteria, in the face of which psychology must make a contribution for the search for an ethical and social project for the construction of the individual and the community. Research and praxis supported by values promoting solidarity and respect for others, and alternative ways of conflict resolution are strategies that must be upheld.

Key words: Social responsibility; Psychology; Violence. 


\section{Resumo}

Neste artigo se há uma análise sobre a responsabilidade social da psicologia frente ao fenômeno da violência. Se parte de seu reconhecimento neste contexto como uma das problemáticas de maior relevância e de saúde pública, que coincide com a valoração que dela fizeram estudos realizados em nível internacional. Se destaca a importância da projeção social e a formação de futuros profissionais desde a universidade, assim como alguns dos estudos sobre as diferentes expressões de violência neste meio. Se assume como importante as recomendações colocadas no relatório mundial da OMS sobre Violência e Saúde e a compreensão da realidade da violência além do âmbito privado e individual no qual sobressaem critérios "curativos" ou legais, para reconhecêla como um fato histórico social frente ao qual a Psicologia deve contribuir com elementos para a busca de um projeto ético/social de construção de sujeito e comunidade. A investigação e práxis sustentada em valores solidários de respeito pelo outro e formas diferentes de solucionar o conflito, serão estratégias que deverão ser sustentadas.

Palavras chave: Responsabilidade social; Psicologia; Violências. 


\section{Introducción}

Los agobiantes problemas que tienen lugar en la sociedad de hoy y afectan de diversas formas a los individuos, son sin duda alguna la conjugación de situaciones y hechos engastados en los procesos históricos y culturales de las sociedades actuales.

Una de tantas problemáticas que afecta la cotidianidad de los colombianos es la violencia. Ésta deteriora permanentemente la calidad de vida de los ciudadanos por los altos niveles de inseguridad que tienen que vivir, las pérdidas materiales, el costo de muchas vidas, las migraciones que provoca y la incidencia que tiene en el desarrollo económico y social del país. Es así como el Plan Nacional de Desarrollo 2006 - 2010, propuesto por el actual Gobierno, señala como uno de sus objetivos centrales la construcción de equidad social a través de diferentes programas. En éstos, se reconoce a la violencia como una de las problemáticas centrales del país a la cual hay que atender.

Sin duda alguna, la aproximación al significado de la violencia demanda la comprensión del marco cultural y social en el que ésta emerge. Es ahí donde los actores sociales construyen su identidad, establecen vínculos y diferentes formas de interacción que median su participación como miembros de cualquier comunidad (Serrano 1996). La búsqueda del entendimiento y la comprensión de los hechos histórico-sociales de los cuales hace parte la violencia y el reconocimiento de su particularidad y peculiaridad genera implicaciones tanto éticas como científicas en el campo de las Ciencias Sociales. Esto supone para la Psicología un acercamiento que va más allá del marco del análisis individual; y significa que deben ser contempladas en la investigación y praxis, condiciones sociales, políticas yeconómicas que inciden en su génesis y en su mantenimiento (Montero, 2003). 
El objetivo de este trabajo es analizar la Responsabilidad Social que desde la universidad tiene la disciplina de la Psicología frente a una realidad que atraviesa las sociedades actuales: la violencia. Para lograrlo, se tomarán inicialmente algunos planteamientos sobre el significado que tiene la Responsabilidad Social en la universidad como un tipo de organización. Su función social es diferente a la de otras organizaciones, e igualmente, se revisarán algunos aspectos que han orientado el quehacer de la Psicología frente a la violencia. Sánchez (2007) entiende la Responsabilidad Social de la educación superior como la correspondencia entre las necesidades sociales e individuales que debe satisfacer y lo que realmente logra. Ello supone una relación entre los procesos de formación del recurso humano y la generación de nuevo conocimiento (objetivos centrales de la educación superior) y las demandas de la sociedad en la que se encuentra inmersa. Implica, entonces, una toma de conciencia frente a sí misma, su entorno y su papel en el entorno. Es decir, se hablaría de Responsabilidad Social a partir del reconocimiento que hace la universidad, del impacto que tienen sus acciones. Este reconocimiento debe ir ligado a preocupaciones de carácter ético (Savater, 1998). Compartiendo el planteamiento de Cortina (2000), quien define la ética como "un tipo de saber, que pretende orientar la acción humana, en un sentido racional" (p. 17); los procesos y acciones que desarrolla la universidad deben fundamentarse en principios y valores que respondan con compromiso y de manera pertinente y eficaz a las necesidades del entorno. En ese mismo sentido Hoyos (1998) concibe la responsabilidad social de manera integral, es decir, es la actuación que implica sensibilidad moral articulada con el cambio social.

Ernst (2008, citado por Mendivelso, 2008), reconoce la responsabilidad que tienen los académicos en la educación de 
las generaciones futuras, cuando afirma que no basta con formar científicos brillantes, sino que es fundamental considerar en la formación la visión ética con énfasis en la responsabilidad; al respecto, este profesor Nobel de Química señala nos debe importar la responsabilidad, porque somos los únicos que podemos decir lo que pensamos... sobre temas importantes. Ciencia y Ética son un matrimonio necesario para este milenio (p. 7).

Tradicionalmente, la universidad ha entendido el ejercicio de la Responsabilidad Social desde la proyección social y la extensión. En ese orden de ideas, el Proyecto Chileno Universidad Construye País (2003) define la Responsabilidad Social "Como la capacidad que tiene la universidad como institución de difundir y poner en práctica un conjunto de principios y valores, por medio de cuatro procesos claves: gestión, docencia, investigación y extensión"(p. 1). Sin embargo, como lo indica Vayaeis (2008), la Universidad en el mundo actual se ve avocada a reflexionar sobre el significado social de la producción de conocimiento, en un momento en el que los desarrollos científicos ocupan un lugar privilegiado en la sociedad. Sánchez (2007), considera que en la sociedad del conocimiento la Responsabilidad Social de la educación superior debe constituirse en un eje estructural, lo que implica no solamente responsabilidad en la producción de conocimiento, sino en la transmisión del mismo y en la formación de ciudadanos responsables (Díaz, 2006).

En este sentido, Bies, Bartunek, Fort y Zald (2007) señalan la necesidad de vincular las instituciones académicas y la investigación que en ellas se realiza a las acciones de Responsabilidad Social de la empresa privada. Esto a través de la conformación de redes académicas comprometidas en mejorar la calidad de vida de las comunidades. 
Desde este planteamiento, el ejercicio de la Responsabilidad Social Universitaria debe articular la complejidad de la realidad y las expectativas de las nuevas generaciones. Sin duda alguna, el reto reside en el fortalecimiento de las relaciones entre la universidad y la sociedad que se posibilita solamente a partir de la reflexión crítica frente al significado social de la producción de conocimiento, que deben hacer conjuntamente los académicos, los estudiantes y la sociedad civil (García, 1997). Particularmente, la Psicología, como disciplina y profesión, no ha sido ajena a este debate. Es así como en Europa, desde la década de los noventa, se planteó la necesidad de integración en la formación, es decir, un currículo que articula los aspectos disciplinares de la Psicología con las necesidades del contexto, enmarcadas en las historias particulares de cada país.

De manera general, se puede identificar una serie de preocupaciones que ha incidido en las propuestas de estudio que se ofrecen. Se mencionan aspectos como el conocimiento fundamental o básico de la disciplina, el desarrollo del conocimiento profesional, la necesidad de la interdisciplinariedad para abordar la realidad, así como la posibilidad de la misma en el ejercicio profesional del psicólogo. En esta misma dirección Ardila (2004), en su artículo La Psicología colombiana en el futuro cercano plantea como características fundamentales para su desarrollo científico, la necesidad de la relevancia social del conocimiento. Además, el trabajo sobre problemas complejos relacionados con el conflicto y la convivencia. Otro de los aspectos considerados como relevantes es el que hace referencia al compromiso y a la Responsabilidad Social que tiene la disciplina, lo que plantea el problema del conocimiento socialmente responsable (Sánchez, 2007). A este respecto, Hoffmann (2008, citado por Mendivelso, 2008) afirma que la responsabi- 
lidad de los científicos no se debe limitar al interés exclusivo por el conocimiento, sino que debe considerar las implicaciones del mismo, lo que define el compromiso que debe existir entre ciencia y ética.

En la Conferencia Mundial de la Educación Superior (1998), realizada en París, se acordaron una serie de aspectos que a manera de acuerdos mínimos debe considerar la universidad para lograr ser responsable socialmente. El primero de ellos se relaciona con la evaluación de la pertinencia. Es decir, la correspondencia que existe entre lo que la sociedad espera de ella y lo que ésta realmente hace. El segundo aspecto mencionado hace referencia a la función de servicio a la sociedad que debe tener desde una aproximación transdisciplinaria e interdisciplinaria frente a la realidad. Este planteamiento es especialmente importante en el contexto latinoamericano y particularmente en un país como Colombia, dada nuestra situación socio-política actual, permeada por condiciones de inequidad. El tercer aspecto mencionado reconoce el compromiso conel desarrollo y fortalecimiento del sistema educativo en términos de formación, diseño de currículos e investigación.

Un último aspecto, señala la responsabilidad de la universidad en la construcción de una sociedad no violenta. Asímismo, en la Conferencia Regional de la Educación Superior en América Latina y el Caribe (CRES), realizada por el Instituto Internacional para la Educación Superior en America Latina y el Caribe, recientemente, en Cartagena (2008) se reivindicó: 
El carácter humanista de la educación superior, en función de la cual ella debe estar orientada a la formación integral de personas ciudadanos y profesionales capaces de abordar con responsabilidad ética, social y ambiental los múltiples retos implicados en el desarrollo endógeno y la integración de nuestros países, y participar activa, crítica constructivamente en la sociedad (p. 9). Además, se señaló la necesidad de impulsar un modelo académico (...) caracterizado por la indagación de los problemas en sus contextos y la producción y transferencia del valor social de los conocimientos (p. 7).

El reconocimiento público y académico del fenómeno de la violencia como una problemática del mundo actual y las implicaciones que de allí se derivan, se ve reflejado en el importante número de estudios que sobre este tema se han realizado. Se indica la necesidad de la comprensión y construcción de posibles alternativas de intervención frente a la misma. La violencia ha pasado por todas las épocas y, sin embargo, conserva el poder de asombro que hay en lo novedoso. Por ello hay que preguntarse: "si nuestra época se distingue por la agudeza y la magnitud de la violencia, o si simplemente tenemos más conciencia de ella, de su carácter irracional y de las injusticias que expresa y produce" (Chesnais, 1992, p. 6).

Los estudios en nuestro medio se han acercado a comprenderla y explicarla desde diferentes perspectivas con variados objetivos, que van desde entenderla como una manifestación de nuestra naturaleza, como un patrón aprendido y mantenido por condiciones del contexto, hasta considerarla como una condición que atraviesa nuestra cultura. Es decir, que forma parte de nuestros procesos de socialización. Adicionalmente, identificar sus diferentes expresiones y contextos en los que aparece, así como el diseño de estra- 
tegias de intervención con el objetivo de generar condiciones que permitan su prevención. Al interior de estas miradas, se conjugan posturas teóricas y metodologías de investigación variada que sin duda alguna han aportado a enriquecer su marco de análisis.

En este sentido, la variabilidad de tópicos estudiados que aportan al compromiso planteado anteriormente, aunque sin llegar a ser exhaustivos, incluyen investigaciones en adolescentes, familias y comunidades.

En el caso de los niños y los adolescentes, la pobreza, las condiciones familiares y de pareja en conflicto y asociadas al consumo de drogas, son valoradas como factores de riesgo con implicaciones importantes en el desarrollo emocional y social. Aspectos que han sido considerados como derechos fundamentales, para los cuales la sociedad deberá velar porque éstos se cumplan. Si bien, en algunos estudios como los de Amar (2000), en los que se ha reconocido que los niños logran superar la adversidad a pesar de estar expuestos a condiciones que no son favorables, existen otros en los que el riesgo está asociado a la tendencia de valorar negativamente a los hijos y ser maltratados físicamente (Klevens, Bayon y Sierra, 2000). Cuevas (2008) llevó a cabo un análisis sobre las consecuencias de la exposición a violencia de niños y adolescentes de colegios de Cali con sus implicaciones para la investigación, la práctica y la política pública. Una de las revisiones más alarmantes sobre esta problemática la llevó a cabo Ramírez (2006) en una publicación, en la cual muestra la magnitud y las consecuencias negativas en las distintas esferas del desarrollo de los niños y niñas, y cómo éste interfiere con la salud mental en la adultez.

En la misma línea de trabajo, Hewitt (2005) encontró en poblaciones similares que los factores cognitivos, atribuciones y 
expectativas son variables determinantes en la co-ocurrencia del maltrato físico a los hijos y el consumo de alcohol. Lo anterior es reforzado por Abello, Amar y Magendzo (2006) al considerar que la restricción, frente a las oportunidades de interacción del niño con unos acontecimientos educativos y familiares, limita su desarrollo cognoscitivo y condiciona su potencial a futuro.

En un país como el nuestro, en el que la violencia se expresa de muchas maneras, la investigación ha privilegiado algunas problemáticas y poblaciones consideradas vulnerables, ocupando un lugar importante el tema del maltrato y abuso en el que cada vez se reportan cifras alarmantes, sin restricción de estrato y género (Velázquez, 2003). Ortiz (1992, citado por Jimeno, 1996) plantea la relación que existe entre la violencia y las características estructurales de la sociedad colombiana. Esta autora señala cómo la familia es parte de un sistema que sostiene y legitima sus prácticas, aunque se modifica, mantiene su idea de comunidad y su noción de dominación y jerarquización. Los resultados de varias de las encuestas realizadas en el país a mujeres en la última década, sustentan esta realidad; muestran, por ejemplo, que entre el 33\% y 37\% de las mujeres comprometidas en algún tipo de unión conyugal han sufrido de violencia verbal, y entre el 19\% y el 39\% han sido víctimas de violencia física (Pineda y Otero, 2004).Según el Informe Violencia contra las Mujeres (2002), realizado por el Banco Mundial, en Colombia, los hombres son los principales perpetradores de la violencia doméstica y en el espacio público sus principales víctimas.

El reconocimiento de la realidad de la violencia como una construcción que se presenta y legítima en la práctica familiar cotidiana, hace necesario generar procesos de reflexión frente a las prácticas de socialización que ocurren en la vida familiar, pues en los proce- 
sos de socialización se expresan las concepciones que una cultura tiene acerca del desarrollo de sus miembros (Rodríguez, 1988). Son numerosos los estudios que señalan cómo las expresiones de violencia se relacionan con las ideas de lo que significa ser mujer u hombre, los derechos atribuidos a cada género y el ejercicio del poder dentro del espacio familiar (Pineda y Otero, 2004; Vaca, Chaparro y Pérez, 2006; Viveros, Olavarria y Fuller 2001).

Berger y Luckman (1986) señalan cómo la cotidianidad es punto de partida y referencia fundamental para la construcción de la identidad. Sin duda alguna, es en la recuperación y comprensión de lo cotidiano donde podremos acceder a resignificar ideas relacionadas con las prácticas violentas que han sido perpetuadas en nuestra sociedad a través de los procesos de socialización (Jimeno, 1996; Parra, González, Moritz, Blandón y Bustamante, 2000). En este mismo sentido, Bolívar y Oviedo (2004) proponen que el mejor camino para la construcción de una cultura de la no-violencia corresponde a la vivencia cotidiana (en el hogar, la escuela y la sociedad) cifrada en la equidad, la democracia participativa, la tolerancia y el respeto por la diferencia. Esta propuesta se deriva de los resultados de su investigación, en la cual encontraron patrones comunes en el trato inadecuado a los niños por parte de los adultos y al flagelo del alcoholismo y otras sustancias.

El interés por ampliar el espectro de posibilidades en el seguimiento a los procesos de socialización en los niños, llevó a Vaca y Romero (2007) a estudiar los significados que construyen los niños alrededor de las propuestas violentas de los videojuegos, y encontraron que éstos se convierten en un agente de socialización importante que contribuye a la perpetuación de interacciones excluyentes. 
En los adultos, la variabilidad en los objetivos de investigación va desde la evaluación de las implicaciones de diferentes formas de violencia en el bienestar de las personas hasta el efecto sobre la salud mental. En estos dos casos, Rodríguez, Díaz, Niño, Samudio y Silva (2005) y Rodríguez (2006) encontraron que si bien el desplazamiento es un factor que contribuye a la crisis, los eventos que lo acompañan se consideran detonantes del desorden de estrés postraumático. Las amenazas de reclutamiento y contra la vida y las torturas se constituyen en eventos de mayor riesgo y a mediano plazo inciden negativamente sobre su nivel de funcionamiento. Esta situación fue valorada también por Palacios (1999) cuando consideró que:

... Colombia es un país que ha sufrido de violencia a lo largo de toda su historia. Esta violencia ha generado un sinnúmero de transformaciones en la vida de todos sus habitantes y una de estas transformaciones la vemos reflejada en las consecuencias psicológicas de las personas desplazadas. Dentro de esta población son los niños y los jóvenes los más afectados, al lado de las mujeres y los ancianos. Este sufrimiento lo vemos reflejado, generalmente en el miedo, las pesadillas, los pensamientos repetitivos sobre los hechos dolorosos, el deseo de huir de lo que nos recuerda los momentos difíciles [...] esto es lo que llamamos estrés postraumático (p. 1).de los conocimientos (p. 7).

Avanzar en esta dirección demanda conocer el fenómeno del desplazamiento, encontrando que múltiples fuentes pueden estar relacionadas. En la investigación realizada por la Organización Internacional para las Migraciones (OIM) y la Pontificia Universidad Javeriana (2002), se muestra cómo el desplazamiento interno 
ha sido provocado por factores complejos y diversos. Se identifica por ejemplo que:

El incremento en el pie de fuerza de la guerrilla y los paramilitares, el mayor control territorial (expansión territorial) de los grupos armados y la riqueza adquirida por estos a través de actividades ilícitas, como el narcotráfico, la extorsión y el secuestro, han generado situaciones de vulnerabilidad para la población civil que han provocado desplazamientos (p. 29).

Si bien los anteriores factores han sido identificados como responsables, son el temor de la sociedad civil y los atentados contra la vida los que animan a los adultos, adolescentes y niños a salir de sus lugares de origen o de vivienda para buscar lugares de seguridad. Esta situación se ha convertido en una amenaza permanente para la sociedad y, a su vez, en una de las experiencias de dolor y sufrimiento humano más significativas (Bello, Mantilla, Mosquera y Camelo, 2000; Bello, 1999).

Otros datos de importancia y que nutren esta revisión son los encontrados por Ramírez (2002) sobre algunas hipótesis que se derivan usando como instrumento la investigación. Esto para interrogar varios discursos sobre la relación de las mujeres con la guerra. Se muestra que en algunos casos la maternidad, como aspecto fundamental, no constituye un obstáculo para modificar su relación con la causa político-militar. Sin embargo, en otros casos, en las mujeres y los hombres los cambios de roles, fruto de las demandas del contexto actual, generan discrepancias y cambios en las formas habituales de relación en el núcleo familiar. A nivel comunitario, se observan dificultades en las nuevas relaciones que deberán establecerse entre la población receptora y la po- 
blación desplazada (Red de Solidaridad Social, 2001). Igualmente, importantes son los hallazgos en relación con la perpetuación de prácticas violentas, evidenciados en la investigación realizada por Sacipa (1999), en los que se muestra cómo hombres y mujeres jóvenes y adultos, de la ciudad de Bogotá, le dan a la violencia el carácter de "natural" al considerarlas como parte de "la naturaleza humana". Lo cual genera, de esta forma, la perpetuación de las relaciones dominador-subordinado, visible muchas de nuestras familias y ámbitos escolares.

Estos resultados reconocen la necesidad de comprender la violencia en toda su complejidad y comparten de alguna manera un planteamiento que va más allá del marco de análisis individual. La perspectiva ecológica aparece como una posibilidad que se convierte en un camino para responder a los interrogantes que nos plantea nuestro compromiso frente a ella (Castellá, 2008). El comprender al sujeto como parte activa de una red de relaciones enmarcadas en un escenario cultural, como lo señala Bronfenbrenner y Morris (1998), solamente en el acercamiento a los diferentes sistemas en los que se desarrolla la actividad humana será posible comprender los fenómenos psicológicos, y reconocer en él el potencial de transformación de su realidad, es un hecho fundamental para orientar nuestro compromiso.

El comprenderla como una realidad que se puede transformar representa para las Ciencias Sociales un compromiso en términos del impacto social del conocimiento. La responsabilidad social se debe orientar a la transformación de esas realidades desde la investigación y la praxis, que, como una posible fuente de conocimiento, representa un camino viable en la construcción de una sociedad equitativa y sostenible (Hoyos y Martínez, 2004). 
Los trabajos realizados por la disciplina de la Psicología, han permitido comprender e identificar factores y condiciones que se relacionan tanto con la generación del fenómeno como con la perpetuación del mismo. De la misma manera, han propuesto formas de intervención contribuidoras a la construcción de realidades alternativas. Sin embargo, hay confrontación a cifras y estadísticas que cada día nos señalan la necesidad de comprometerse aún más con esta realidad (Escobedo, 2007).

En este sentido, las recomendaciones de Krug, Dahlberg, Mercy, Zwi, y Wilson (2003), planteadas en el Informe Mundial sobre la Violencia y la Salud de la OPS, señalan una serie de aspectos que deben ser considerados para enfrentar la violencia. Toman como punto de partida que este fenómeno puede predecirse y prevenirse, dado que las investigaciones a la fecha han identificado factores tanto micro como macro que se relacionan con su aparición.

De la misma manera, insisten en la necesidad de continuar la investigación con miras a comprender su naturaleza desde las perspectivas cualitativas y cuantitativas y retroalimentar los datos existentes, de tal manera que nutran la formulación de estrategias y políticas frente a esta realidad. Asumida por el informe como un problema de salud pública.

La comprensión del contexto y, por tanto, la particularidad del fenómeno debe ser visible. Camacho (2002) señala que en la presencia de la violencia hay circunstancias históricas específicas que demandan para su explicación el reconocimiento de esas especificidades. Sin duda alguna, existen creencias en algunas sociedades que legitiman su expresión, que deben ser consideradas a la hora de pensar en propuestas de intervención. 
Por último, enfatizan en la necesidad de fortalecer y construir redes de información, que permitan superar la fragmentación y a su vez, una mayor difusión de los resultados de las investigaciones que se realizan en este campo. Recomendaciones que harían realidad el trabajo sobre la prevención.

Se considera que el compromiso para la Psicología se encuentra tanto en los procesos de formación de los futuros psicólogos, como en el ejercicio de la investigación y la praxis que se realiza. Se comparte y se considera vigente el planteamiento realizado por Díaz-Aguado (1999), en relación con el papel de la Psicología en la lucha contra la violencia. Allí señala una serie de principios que deben orientar nuestra acción. Estos se sitúan desde la responsabilidad en hacerla visible resignificando el carácter de "natural" con el que es asumida por muchas de nuestras poblaciones, hasta el compromiso de develar en toda su complejidad su origen. Sin dejar de lado la necesidad de intervenir en los diferentes sistemas en los que se expresa.

El reto se plantea en situar a la violencia más allá del ámbito privado e individual en el que priman criterios "curativos" o legales. Por supuesto son importantes, y reconocerla como un hecho histórico social frente a la cual la Psicología debe aportar elementos para la búsqueda, de un proyecto ético/social de construcción de sujeto y comunidad. Esto a través de la investigación y praxis, soportada en valores solidarios de respeto por el otro y formas diferentes de solucionar el conflicto. 


\section{Referencias}

Abello, R., Amar, J. y Magendzo, S. (2006). Toma de perspectiva de niños pobres en Colombia. Infancia, Adolescencia y Familia, 1, $189-204$.

Amar, J. (2000). Niños invulnerables. Factores cotidianos de protección que favorecen el desarrollo de los niños que viven en contextos de pobreza. Psicología desde el Caribe, 5, 96-126.

Ardila, R. (2004). La Psicología colombiana en el futuro cercano. Revista de Estudios Especiales, 18, 142-144.

Banco Mundial (2002). Informe Violencia contra las mujeres. Recuperado 12, junio, 2008 de http://www.unifem.org

Berger, P. y Luckman, T. (1986). La construcción social de la realidad. Buenos Aires: Amorrortu.

Bies, R., Bartunek, J., Fort, T. y Zald M. (2007). Corporations as social change agents: individual, interpersonal, institutional, and environmental dynamics. Academy of Management Review, 32, 3, 788-793.

Bello, M. (1999). Las familias desplazadas por la violencia. Un tránsito abrupto del campo a la ciudad. Revista del Departamento de Trabajo Social, 2, 25-38.

Bello, M., Mantilla L., Mosquera, C. y Camelo, E. (2000). Relatos de la violencia. Universidad Nacional de Colombia. Bogotá.

Bolívar, C. y Oviedo, M. (2004). Representaciones sociales infantiles de la convivencia y el Conflicto. Grupo de Investigación 
Crecer. Universidad Surcolombiana, Facultad de Salud, Programa de Psicología. Neiva.

Bronfenbrenner, U. y Morris, P.A. (1998). The Ecology of Developmental Processes. En: W. Dannon y R.M. Lerner (Eds.), Hand Book of Child Psychology, Nueva York: (pp. 993-1028) John Wiley \& Sons, Inc.

Camacho, A. (2002). El ayer y el hoy de la violencia en Colombia: continuidades y discontinuidades. Estudios. Recuperado 30, Junio, 2009 de http://www.labla.org

Castellá, J. (2008). Enfoques conceptuales y técnicos en Psicología Comunitaria. Buenos Aires: Paidós.

Chesnais, J.C. (1992). Historia de la violencia: el homicidio y el suicidio a través de la historia. Revista Internacional de Ciencias Sociales, 8, 23-32.

Conferencia Mundial de la Educación Superior. (1998). Declaración mundial sobre la educación superior en el Siglo XXI: visión y acción. Recuperado 30, Junio, 2009 de http://www.unesco.org

Cortina, A. (2000). Ética de la empresa. Valladolid: Trota.

Cuevas, M.C. (2008). Exposición a violencia de niños y adolescentes de colegios de Cali: implicaciones para la investigación, la práctica y la política. Cátedra Mercedes Rodrigo. Pontificia Universidad Javeriana-Cali.

Díaz-Aguado, M. (1999). El papel de la Psicología en la lucha contra la violencia. Recuperado 12, Junio, 2008 de http://www. contrilinterno.udea.edu 
Díaz, G. (2006). Origen e idea de universidad. Universidad de Ibagué-Coruniversitaria Foro Responsabilidad Social: una mirada desde la universidad.

Escobedo, R. (2007). Rompiendo el silencio, mujer víctima y victimaria. Secretaría Distrital de Gobierno. Bogotá.

García, M. (1997). Elementos para la construcción de una Ética Civil en Colombia. Memorias Seminario Ética Civil y Convivencia Ciudadana. Pontificia Universidad Javeriana. Cali.

Hewitt, N. (2005). Factores cognoscitivos de los padres asociados a la co-ocurrencia del consumo de alcohol y el maltrato físico a los hijos. Acta Colombiana de Psicología, 13, 7-34.

Hoyos, G. (1998). Ética Ciudadana y Derechos Humanos de los Niños: una contribución a la paz. Bogotá: Magisterio.

Hoyos, G. y Martínez, M. (2004). Qué significa educar en valores. Buenos Aires: Octaedro Edición.

Instituto Internacional para la Educación Superior en América Latina y el Caribe, Iesalc. (2008). Declaración de la Conferencia Regional de la Educación Superior en América Latina y el Caribe CRES. Recuperado 30, Octubre, 2008 de http://www.cres2008. org/es/index.php

Jimeno, M. (1996). Las sombras arbitrarias. Bogotá: EUN.

Klevens, J., Bayón, M.C. y Sierra, M. (2000). Risk Factors and Context of Men Who Physically Abuse in Bogotá, Colombia. Journal Child Abuse \& Neglect, 24, 3, 323-332. 
Krug, E. G., Dahlberg, L.L., Mercy, J. A., Zwi, A.B. y Wilson, A. (2003). Cómo proceder: recomendaciones. En E.G. Krug,

L.L. Dahlberg, J.A. Mercy, A. B. Zwi, y R. Lozano (Eds), Informe mundial sobre la violencia y la salud. Organización Panamericana de la Salud. Washington. Recuperado 20, noviembre, 2008 de http://www.mex.opsoms.org/contenido/ cd_lencia/documentos/ informemundial_completo.pdf

Mendivelso, N. (2008). Ciencia y Ética: Matrimonio necesario para este milenio. UN Periódico, 118, 7.

Montero, M. (2003). Teoría y Práctica de la Psicología Comunitaria. Buenos Aires: Paidós.

Organización Internacional para las Migraciones y Pontificia Universidad Javeriana. (2002) Desplazamiento interno y atención psicosocial: el reto de reinventar la vida, estado del arte. Bogotá: Servigraphic.

Palacios, J. (1999). Estrés postraumático y Resistencia psicológica en jóvenes desplazados. Centro de Investigación en DesarroIlo Humano (Cidhum) y el laboratorio de Psicología del DesarroIlo, de la Universidad de París Nanterre.

Parra, R., González, A., Moritz, O., Blandón, A. y Bustamante, R. (2000). La Escuela Violenta. Bogotá: TM Editores.

Plan Nacional de Desarrollo 2006-2010: ¿desarrollo para todos? Departamento Nacional de Planeación. (2008). Recuperado 20, noviembre, 2008 de www.dnp.gov.co/ PortalWeb/PND/ PND20062010/tabid/65/ Default.aspx 
Pineda, J. y Otero, L. (2004). Género violencia intrafamiliar e intervención pública en Colombia. Revista de Estudios Sociales, 17, 24-36.

Ramírez, M. (2002). Las mujeres y la guerra. Psicología desde el Caribe, 9, 90-124.

Ramírez, C. (2006). Maltrato en los niños y las niñas en Colombia. Revista Infancia, Adolescencia y Familia, 1, 2, 287-301.

Red de Solidaridad Social. (2001). Atención a la población desplazada por la violencia en Colombia. Presidencia de la República.

Rodríguez, A. (1988). Psicología Política. Buenos Aires: Pirámide.

Rodríguez, M.C., Díaz, P., Niño, S., Samudio, M. y Silva, M. (2005). El desplazamiento como generador de crisis: un estudio en adultos y adolescentes. Terapia Psicológica, 23, 33-43.

Rodríguez, M.C. (2006). Programa de grupo estructurado en estrategias de afrontamiento Para DSPT en adultos y adolescentes en situación de desplazamiento. Univérsitas Psicológica, 5, 2, 259-274.

Sacipa, S. (1999). Anclados en las dinámicas de las violencias. Pontificia Universidad Javeriana. Facultad de Psicología. Bogotá.

Sánchez, L. (2007) La Responsabilidad Social Universitaria. Recuperado 12, Junio, 2008 de http://www.contrilinterno.udea.edu

Savater, F. (1998). La dimensión ética de la empresa. Bogotá: Siglo del Hombre Editores.

Serrano, J. (1996). La Psicología Cultural como psicología crítico interpretativa. En Psicología discursos y poder. Madrid: Vuisor. 
Universidad Construye País. (2003). Recuperado 23, junio, 2009 de http://www. construyepais.cl

Vaca, P. y Romero, D. (2007). Construcción de significados frente a los contenidos violentos de los video juegos en niños de 11 a 14 años. Acta Colombiana de Psicología, 10, 1, 35-48.

Vaca, P., Chaparro, B. y Pérez, N. (2006). Representaciones sociales acerca de la identidad de género de una mujer que emplea la violencia en la solución de conflictos. Psicología desde el Caribe, $18,37-45$.

Viveros, M., Olavarria, J. y Fuller, N. (2001). Hombres e identidades de género. Universidad Nacional de Colombia: Bogotá.

Velázquez, S. (2003). Violencias cotidianas, violencia de género. Buenos Aires: Paidós.

Vayaeis, L. (2008). Responsabilidad Social. Recuperado 12, junio, 2008 de http://www. contrilinterno.udea.edu 